\title{
Deposição de pontas de pulverização AXI 11002 e JA-2 em diferentes condições operacionais
}

\author{
Deposition of AXI 11002 and JA-2 nozzles in different operational conditions
}

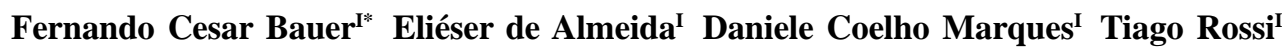 \\ Francisco de Assis Rolim Pereira ${ }^{\mathrm{I}}$
}

\section{RESUMO}

Este trabalho teve como objetivo avaliar a deposição da pulverização das pontas jato plano AXI 11002 e jato cônico vazio JA-2 utilizadas com duas pressões, com e sem a utilização da assistência de ar em cultura da soja. Para isso, foram avaliadas a distribuição de pontas AXI 11002 nas pressões de 300 e $400 \mathrm{kPa}$ e de pontas JA-2 nas pressões de 400 e $600 \mathrm{kPa}$, todos com e sem assistência de ar. Os tratamentos foram dispostos em blocos ao acaso, com duas repetições em diferentes estádios da cultura. A calda foi preparada utilizando-se o corante Azul Brilhante (FDC-1) a 0,3\% como traçador. As aplicações foram realizadas por meio de um pulverizador hidráulico Modelo Falcon Vortex. Os resultados mostraram que a assistência de ar junto à barra de pulverização, de maneira geral, não influenciou significativamente a deposição total da calda pulverizada na planta, mas proporcionou uma melhor penetração nas porções inferiores das plantas de soja.

Palavras-chave: tecnologia de aplicação, soja, assistência de ar.

\section{ABSTRACT}

The aim of this research was to evaluate the deposition obtained with flat fan nozzle AXI 11002 and hollow cone JA-2 operated in two pressure and with and without air assistance in soybean crop. The flat fan nozzles at 300 and $400 \mathrm{kPa}$ and hollow cone at 400 and $600 \mathrm{kPa}$, with and without air assistance were evaluated. The experimental design were randomized blocks and two repetitions in different times of the plants. The solution tracer was composed of Brilliant Blue (FDC-1) at $0.3 \%$. The applications were executed by air assisted boom sprayer, Falcon Vortex Model. The results showed that the air assistance doesn't influence on the total deposition in the plant, but it provided a better penetration and more deposition in the botton of the plants.

Key words: application technology, soybean crop, air assisted.

\section{INTRODUÇÃO}

Os agrotóxicos, embora desempenhem papel importante no sistema de produção agrícola vigente, têm sido alvo de crescente preocupação, em virtude de seu potencial de risco ambiental (BARCELLOS et al., 1998). Um dos fatores importantes para o sucesso das aplicações é o estudo das relações entre o tipo de alvo a ser atingido, a forma de ação do defensivo e a técnica utilizada para a aplicação (ANTUNIASSI, 2004). A seleção das pontas de pulverização serve para adequar o pulverizador ao tipo de aplicação que será realizada, buscando sempre otimizar o resultado biológico e a capacidade da máquina (BOLLER, 2006).

Durante a pulverização de um herbicida ou outro defensivo agrícola, parte da quantidade aplicada não atinge o alvo desejado. Por isso, o aumento no custo desses produtos, da mão-de-obra e de energia despendida e a preocupação cada vez mais crescente em relação à poluição ambiental têm realçado a necessidade de uma tecnologia mais apurada para a colocação do produto químico no alvo (MATUO, 1998). A avaliação dos depósitos da calda aplicada em alvos naturais ou artificiais é o método mais representativo para entender diversos aspectos relacionados à pulverização de defensivos agrícolas. No entanto, os custos dessas pesquisas podem ser um fator limitante na condução dos estudos, quando as determinações são realizadas utilizando-se os próprios defensivos agrícolas, visto que necessitam de reagentes

${ }^{\mathrm{I} C u r s o ~ d e ~ A g r o n o m i a, ~ U n i v e r s i d a d e ~ p a r a ~ o ~ D e s e n v o l v i m e n t o ~ d o ~ E s t a d o ~ e ~ d a ~ R e g i a ̃ o ~ d o ~ P a n t a n a l ~(U N I D E R P), ~ C a m p u s ~ I I I, ~ C P ~}$ 2153, 79037-280, Campo Grande, MS, Brasil. E-mail: fcbauer@mail.uniderp.br. *Autor para correspondência. 
apropriados, equipamentos sofisticados e pessoas treinadas para a realização das análises (MATUO, 1988). Assim, o uso de substância traçadora se torna muito atrativo, em virtude da facilidade de visualização ou remoção das folhas ou alvos coletores diretamente pela utilização de água.

O presente trabalho teve como objetivo avaliar, utilizando substância traçadora, a deposição da pulverização na planta, obtidas por pontas de pulverização hidráulicas de jato plano AXI 11002 e jato cônico vazio JA-2, submetido a duas pressões e presença de assistência de ar.

\section{MATERIAL E MÉTODOS}

O experimento foi instalado e conduzido no campo experimental da Fazenda Escola Três Barras, que pertence a Universidade para o Desenvolvimento do Estado e da Região do Pantanal (UNIDERP), Campus de Campo Grande - MS.

Os tratamentos foram constituídos por dois tipos de pontas de pulverização: AXI 11002 nas pressões de 300 e $400 \mathrm{kPa}$ e de pontas JA-2 nas pressões de 400 e $600 \mathrm{kPa}$, sendo que cada tipo de ponta e pressão foram também avaliadas com e sem assistência de ar (Tabela 1). O corante Azul Brilhante (FDC-1) foi utilizado misturado a água da calda, na concentração de $0,3 \%$. Segundo PALLADINI (2000), esse corante não interfere nas características físicas da calda, podendo ser utilizado como traçador para simulação da aplicação de produtos fitossanitários.

O delineamento experimental foi em blocos ao acaso, com duas repetições em diferentes estádios da cultura. As parcelas mediram 8 x 14m com uma área de $8 \mathrm{~m}$ entre cada parcela destinada para manobra do conjunto trator-pulverizador.

Os tratamentos foram testados em cultura da soja, cultivar "Coodetec 211", semeadas em 09/12/ 05 , com $0,45 \mathrm{~m}$ de espaçamento entre as linhas de plantio e adubação de plantio com $300 \mathrm{~kg} \mathrm{ha}^{-1}$ do formulado 02 $30-20$.
As aplicações foram realizadas com auxilio de um trator Massey Ferguson 297 trabalhando na primeira marcha em 1.700rpm, resultando em velocidade de $6,2 \mathrm{~km} \mathrm{~h}^{-1}$. O pulverizador utilizado foi um Modelo Falcon Vortex fabricado por Máquinas Agrícolas Jacto S.A., equipado com barras de $14 \mathrm{~m}$, com assistência de ar e bicos espaçados em $0,5 \mathrm{~m}$, operando a $0,5 \mathrm{~m}$ acima das plantas.

A primeira aplicação foi realizada em 13/02/ 06 , das $8 \mathrm{~h} 30 \mathrm{~min}$ às $9 \mathrm{~h} 30 \mathrm{~min}$. Durante a aplicação dos tratamentos, a temperatura média foi $33^{\circ} \mathrm{C}$ e a umidade relativa do ar de $58 \%$.

A segunda aplicação foi no dia 14/03/06, das $9 \mathrm{~h}$ às $10 \mathrm{~h}$. Durante a aplicação dos tratamentos a temperatura média foi de $27,4^{\circ} \mathrm{C}$, a umidade relativa do ar em 74\%. Segundo RITCHIE et al. (1982), citado por EMBRAPA (2005), a cultura se encontrava no estádio R2 e R5.2 durante a primeira e segunda aplicações, respectivamente.

Após a aplicação foram coletadas duas amostras de 15 folíolos por tratamento, sendo 15 da parte superior e 15 da parte inferior das plantas, retirados aleatoriamente em uma área de 3 x 10m dentro da área experimental, desprezando-se as bordaduras. Os folíolos amostrados foram colocados, individualmente, em saco plástico identificado e armazenado em caixa térmica ainda no campo. Após o término da coleta, as amostras foram transferidas para um refrigerador, onde permaneceram por período máximo de três dias. Após a coleta, os folíolos foram lavados, dentro dos respectivos sacos plásticos, em $10 \mathrm{ml}$ de água destilada, por meio da agitação do saco para extrair o corante retido na superfície foliar. Após a lavagem, a solução resultante foi depositada em recipiente de vidro identificado, constituindo assim as amostras para análise. Após a lavagem determinou-se a área foliar de cada folíolo, por meio de medidor de área foliar Modelo LI-300 (Licor Inst. Inc., USA). A quantificação do corante presente nas amostras foi feita por meio de leitura em espectrofotômetro FEMTO, Modelo 700 PLUS, determinando-se a absorbância no comprimento de onda de $630 \mathrm{~nm}$.

Tabela 1 - Características dos tratamentos utilizados em aplicações na cultura da soja. Campo Grande, 2006.

\begin{tabular}{clcccc}
\hline Tratamento & Ponta & Assistência de ar & Pressão (kPa) & Tamanho de gotas (DMV) & Volume de aplicação (L ha $\left.{ }^{-1}\right)$ \\
\hline 1 & AXI 11002 & sem & 300 & 152 & 166,5 \\
2 & AXI 11002 & com & 300 & 139 & 166,5 \\
3 & AXI 11002 & sem & 400 & 139 & 191,6 \\
4 & AXI 11002 & com & 400 & 153 & 128,0 \\
5 & JA-2 & sem & 400 & 153 & 128,0 \\
6 & JA-2 & com & 400 & 148 & 147,1 \\
7 & JA-2 & com & 600 & 148 & 147,1 \\
\hline
\end{tabular}

Ciência Rural, v.38, n.6, set, 2008. 
Os valores de absorbância foram transformados em volume, permitindo a associação da área foliar de cada folíolo ao volume nele depositado ( $\mu \mathrm{L}$ de calda $\mathrm{cm}^{-2}$ ), conforme NEGRISOLE et al. (2002). Os dados foram submetidos à análise de variância pelo teste $\mathrm{F}$ e as médias comparadas pelo teste de Tukey em nível de $5 \%$ de probabilidade.

\section{RESULTADOS E DISCUSSÃO}

A influência da assistência de ar e pressão de trabalho, para as diferentes pontas, na deposição total da calda, independente da posição de amostragem, em plantas de soja em dois estádios de desenvolvimento, podem ser observadas na tabela 2 .

Verifica-se que a deposição da calda aplicada nas plantas de soja, de modo geral, não teve aumento significativo com a assistência de ar junto à barra de pulverização, para as diferentes pontas de pulverização com as plantas no estádio de desenvolvimento R2. Entretanto, no estádio de desenvolvimento R5.2, as pontas AXI 11002 com assistência de ar, apresentaram níveis significativamente maiores de depósito de calda em comparação com a ausência desse fator, para as pressões de 300 e $400 \mathrm{kPa}$. A ponta JA-2 na pressão de $600 \mathrm{kPa}$, no estádio R5.2, também teve aumento significativo na deposição da calda com assistência de ar apresentando níveis de depósitos estatisticamente iguais aos obtidos com a ponta AXI 11002 em presença e ausência de ar quando à pressão de $300 \mathrm{kPa} \mathrm{e}$, para a pressão de $400 \mathrm{kPa}$, na presença de ar. Essa constatação também foi feita por BAUER \& RAETANO (2001), em feijoeiro. Ao avaliar o efeito da assistência de ar em cultura de soja, no estádio de desenvolvimento R6, BAUER \& RAETANO (2000) também observaram aumento na deposição quando a assistência de ar foi utilizada.

Volumes maiores de calda proporcionaram níveis significativamente maiores de depósito na cultura da soja nos diferentes estádios de desenvolvimento para as pontas JA-2. Entretanto, para a ponta AXI 11002, o volume de calda só teve diferença na presença de ar para o estádio de desenvolvimento $\mathrm{R} 2$.

Os valores médios dos depósitos com pontas de pulverização trabalhando em diferentes condições operacionais, quando comparados em cada posição de amostragem na cultura da soja, em diferentes estádios de desenvolvimento, estão descritos na tabela 3 .

Ao analisar os valores médios dos depósitos de calda nos folíolos da parte inferior da planta, no estádio de desenvolvimento R2, percebe-se que, para ambas as pontas, houve diferença somente quando as maiores pressões foram usadas. Nesse mesmo estádio de desenvolvimento e mesma posição de amostragem, houve maiores níveis de depósitos em todas as aplicações com assistência de ar, embora os valores não tenham sido significativamente diferentes. Entretanto, esse comportamento não ocorreu nas amostragens da deposição na parte superior das plantas, ocasião em que o uso da assistência de ar fez com que os depósitos fossem inferiores à aplicação sem o ar.

Esse comportamento, para esse estádio de desenvolvimento da cultura, indica possível interação entre a assistência de ar e o tamanho das gotas, em que essa interação se torna positiva com a diminuição do tamanho das gotas, melhorando a penetração da pulverização.

Resultados semelhantes de produtividade de grãos foram obtidos aplicando-se fungicida sistêmico com diferentes tecnologias na cultura da soja, indicando haver possibilidade de se dar preferência àquelas com menor risco de deriva (ANTUNIASSI et al., 2004). Portanto, com base nessas informações, a

Tabela 2 - Valores totais médios de depósitos $(\mu \mathrm{L})$ em plantas de soja, em diferentes estádios de desenvolvimento, após pulverização de calda em presença e ausência da assistência de ar junto à barra de pulverização. Campo Grande, 2006.

\begin{tabular}{|c|c|c|c|c|c|}
\hline \multirow{2}{*}{ Ponta de pulverização } & \multirow{2}{*}{ Assistência de ar } & \multirow{2}{*}{ Pressão ( kPa ) } & \multirow{2}{*}{ Volume de aplicação $\left(\mathrm{L} \mathrm{ha}^{-1}\right)$} & \multicolumn{2}{|c|}{-------Deposição total $(\mu \mathrm{L})$------- } \\
\hline & & & & Estádio R2 & Estádio R5.2 \\
\hline AXI 11002 & sem & 300 & 166,5 & $0,2141 \mathrm{ab}$ & $0,2407 \mathrm{bc}$ \\
\hline AXI 11002 & com & 300 & 166,5 & $0,1965 \mathrm{bcd}$ & $0,2823 \mathrm{a}$ \\
\hline AXI 11002 & sem & 400 & 191,6 & $0,2095 \mathrm{a}$ & $0,2183 \mathrm{c}$ \\
\hline AXI 11002 & com & 400 & 191,6 & $0,2253 \mathrm{a}$ & $0,2691 \mathrm{a}$ \\
\hline JA-2 & sem & 400 & 128,0 & $0,1925 \mathrm{~cd}$ & $0,2298 \mathrm{c}$ \\
\hline JA-2 & com & 400 & 128,0 & $0,1800 \mathrm{~d}$ & $0,1602 \mathrm{~d}$ \\
\hline JA-2 & sem & 600 & 147,1 & $0,2141 \mathrm{ab}$ & $0,1567 \mathrm{~d}$ \\
\hline JA-2 & com & 600 & 147,1 & $0,2119 \mathrm{a}$ & $0,2645 \mathrm{ab}$ \\
\hline
\end{tabular}

*Valores, na coluna, seguidos de mesma letra minúscula não diferem entre si pelo Teste de Tukey a 5\% de probabilidade de erro.

Ciência Rural, v.38, n.6, set, 2008. 
Tabela 3 - Valores médios de depósitos $(\mu \mathrm{L})$ em diferentes alturas de plantas de soja após pulverização de calda em presença e ausência da assistência de ar junto à barra de pulverização. Campo Grande, 2006.

\begin{tabular}{|c|c|c|c|c|c|c|}
\hline \multirow{3}{*}{ Ponta de pulverização } & \multirow{3}{*}{ Assistência de ar } & \multirow{3}{*}{ Pressão $(\mathrm{kPa})$} & \multicolumn{2}{|c|}{------Estádio R2------ } & \multicolumn{2}{|c|}{------Estádio R5.2------ } \\
\hline & & & -------- & ----Posição & stragem-- & ------ \\
\hline & & & Inferior & Superior & In ferior & Superior \\
\hline AXI 11002 & sem & 300 & $0,0640 \mathrm{ab}$ & $0,1500 \mathrm{abc}$ & $0,0419 b c$ & $0,1988 b$ \\
\hline AXI 11002 & com & 300 & $0,0646 \mathrm{ab}$ & $0,1319 \mathrm{abc}$ & $0,0484 \mathrm{~b}$ & $0,2731 \mathrm{a}$ \\
\hline AXI 11002 & sem & 400 & $0,0428 \mathrm{c}$ & $0,1666 \mathrm{a}$ & $0,0352 \mathrm{c}$ & $0,1831 b$ \\
\hline AXI 11002 & com & 400 & $0,0714 \mathrm{ab}$ & $0,1539 \mathrm{abc}$ & $0,0540 \mathrm{ab}$ & $0,2151 b$ \\
\hline JA-2 & sem & 400 & $0,0453 \mathrm{c}$ & $0,1472 \mathrm{abc}$ & $0,0493 \mathrm{~b}$ & $0,1805 b$ \\
\hline JA-2 & com & 400 & $0,0593 \mathrm{bc}$ & $0,1208 \mathrm{c}$ & $0,0421 b c$ & $0,1257 \mathrm{c}$ \\
\hline JA-2 & sem & 600 & $0,0536 \mathrm{bc}$ & $0,1606 \mathrm{ab}$ & $0,0420 \mathrm{bc}$ & $0,1147 \mathrm{c}$ \\
\hline JA-2 & com & 600 & $0,0782 \mathrm{a}$ & $0,1246 \mathrm{bc}$ & 0,0629 a & $0,2016 b$ \\
\hline
\end{tabular}

*Valores, na coluna, seguidos de mesma letra minúscula não diferem entre si pelo Teste de Tukey a $5 \%$ de probabilidade de erro.

melhor opção para aplicação, visando as partes mais baixas das plantas de soja, será a ponta de jato plano, que produz gotas de maiores dimensões em relação à ponta de jato cônico vazio.

O aumento do volume de calda e pressão, aliado à assistência de ar, proporcionou níveis de deposição maior nas folhas de soja da parte mais baixa da planta para as pontas JA-2. A deposição nos folíolos da parte superior das plantas, no estádio R2, não mostrou diferença significativa entre as pontas utilizadas no tratamento.

Os resultados obtidos na parte inferior das plantas no estádio de desenvolvimento R5.2 foram semelhantes aos do estádio R2. Porém, com volume de depósitos menores em quase todos os tratamentos. Os valores médios dos depósitos na parte inferior das plantas foram significativamente superiores para a ponta AXI 11002 na pressão de $400 \mathrm{kPa}$ com assistência de ar, contudo, não foram observadas diferenças significativas a $300 \mathrm{kPa}$, tanto na presença quanto na ausência da assistência de ar. Esse comportamento também foi observado com a ponta JA-2 na presença e ausência de assistência de ar. Essa constatação, novamente, indica interação entre a assistência de ar e o tamanho das gotas, pois, assim como em R5.2, maiores deposições foram obtidas com o uso da assistência de ar associada a elevação da pressão. Essa constatação assume destacada importância nos casos em que há necessidade de maior penetração da calda no interior da cultura, como é o caso de aplicações visando o controle da ferrugem da soja.

As pontas JA-2 obtiveram melhores índices de deposição sobre as folhas inferiores quando se aumentou o volume e a pressão na presença da assistência de ar. Nos folíolos da parte superior, as pontas AXI 11002 e a pressão de $300 \mathrm{kPa}$ com assistência de ar foram as que apresentaram níveis de depósitos significativamente superiores.

\section{CONCLUSÕES}

A deposição total da pulverização nas plantas de soja em estádio de desenvolvimento R2 não foi influenciada significativamente pela assistência de ar, diferente do que ocorreu no estádio R5.2. Para as pontas JA-2, maior volume de calda aliado à assistência de ar proporcionou maiores depósitos na parte inferior das plantas de soja em R2 e R5.2.

\section{REFERÊNCIAS}

ANTUNIASSI, U.R. Tecnologia de aplicação de defensivos. Rondonópolis: Fundação de Apoio a Pesquisa Agropecuária de Mato Grosso - Fundação MT, 2004. N.8, p.165-177. (Boletim Técnico de Soja, 2004).

ANTUNIASSI, U.R. et al. Avaliação da cobertura de folhas de soja em aplicação terrestre com diferentes tipos de pontas. In: SIMPÓSIO INTERNACIONAL DE TECNOLOGIA DE APLICAÇÃO DE AGROTÓXICOS, 3., 2004, Botucatu. Anais... Botucatu: FEPAF, 2004. 4p. CD-ROM.

BARCELLOS, L.C. et al. Estudo sobre a penetração de gotas de pulverização no dossel da cultura da soja [Glycine max (L.) Merrill]. Engenharia na Agricultura, v.6, p.81-94, 1998.

BAUER, F.C.; RAETANO, C.G. Influencia da assistência de ar na barra de pulverização na deposição e penetração da calda em folíolos de feijoeiro. In: SIMPÓSIO INTERNACIONAL DE TECNOLOGIA DE APLICAÇÃO DE AGROTÓXICOS: EFICIÊNCIA, ECONOMIA E PRESERVAÇÃO DA SAÚDE HUMANA E DO AMBIENTE, 2., Jundiaí, 2001. Anais... Jundiaí: Instituto Agronômico, Centro de Mecanização e Automação Agrícola, 2001. 6p. 
Acesso em: 07 fev. 2006. On line. Disponível em: http:// www.iac.br/ cma/Sintag/num11a_1a.PDF.

BAUER, F.C.; RAETANO, C.G. Assistência de ar na deposição e perdas de produtos fitossanitários em pulverizações na cultura da soja. Scientia Agrícola, v.57, n.2, p.271-276, 2000.

BOLLER, W. Parâmetros técnicos para seleção de pontas Tecnologia de Aplicação de Defensivos Agrícolas. Passo Fundo: Plantio Direto Eventos, 2006. p.43. (Atualidades Técnicas, 2).

EMPRESA BRASILEIRA DE PESQUISA AGROPECUARIA EMBRAPA. Tecnologia de produção de soja: região central do Brasil 2006. Londrina, 2005. p.220. (Sistema de produção, 9).

MATUO, T. Desenvolvimento de um pulverizador intermitente operado fotoeletricamente para tratamento de pomares de citros. 1988. 167f. Tese (Livre Docência) Universidade Estadual Paulista. Jaboticabal.
MATUO, T. Fundamentos da tecnologia de aplicação de agrotóxicos. In:GUEDES, J.V.C.; DORNELLES, S.H.B. (Eds.). Tecnologia e segurança na aplicação de agrotóxico: novas tecnologias. Santa Maria: Sociedade de Agronomia de Santa Maria, 1998. p.95-103.

NEGRISOLI, E. et al. Depósitos unitários de calda de pulverização com e sem surfatantes em plantas de Salvinia molesta. Planta Daninha. v.20, p.51-56, 2002, Edição Especial.

PALLADINI, L.A. Metodologia para a avaliação da deposição em pulverização. 2000. 111f. Tese (Doutorado em Agronomia/Proteção de Plantas) - Universidade Estadual Paulista, Botucatu.

RITCHIE, S. et al. How a soybean plant develops. Ames: Iowa State University of Science and Technology, Coop Ext Serv, 1982. 20p. (Special Report, 53). 